\title{
Factores climáticos que controlan el crecimiento radial y la formación de fluctuaciones de densidad en la madera de Austrocedrus chilensis en Valdivia, Chile
}

\author{
Climatic factors controlling radial growth and formation of wood density fluctuations \\ in Austrocedrus chilensis in Valdivia, Chile
}

\author{
Vicente Rozas ${ }^{\text {a*, }}$, Carlos Le Quesne ${ }^{\text {a }}$, Moisés Rojas-Badilla ${ }^{\text {a }}$ \\ *Autor de correspondencia: ${ }^{\text {a }}$ Universidad Austral de Chile, Facultad de Ciencias Forestales y Recursos Naturales, \\ Laboratorio de Dendrocronología y Cambio Global, Casilla 567, Valdivia, Chile, tel.: 56-63-2444347, vicenterozas@gmail.com
}

\begin{abstract}
SUMMARY
Tree-ring widths and intrannual wood density fluctuations (IADFs) are sound proxies of the environmental factors that control the growth of forest tree species. In this work we evaluated the impact of climate on radial growth and IADFs formation in an Austrocedrus chilensis plantation in Valdivia, Chile. We calculated mean tree-ring chronology and the annual distribution of IADFs frequency from 20 trees, and we studied their relationships with local precipitation, temperature, and large-scale El Niño-Southern Oscillation (ENSO) and Antarctic Oscillation (AO) climatic teleconnections in the period 1975-2010. We used correlation and multiple linear regression analyses to assess the climate-growth relationships and $t$ test for means comparison to check for differences between years with and without IADFs. We observed an inverse relationship of growth with previous summer precipitation and maximum temperature during the growing period, which suggests physiological stress for trees caused by water surplus and warm summer conditions. Years with maximum IADFs frequency were those coinciding with El Niño events, which caused dry and warm summer conditions in the study area, followed by rainy conditions in September. We did not find statistically significant relationships with AO. The interaction between local climatic conditions and ENSO variation controlled IADFs formation in the wood of $A$. chilensis in Valdivia.
\end{abstract}

Key words: Valdivian climate, wood anatomy, dendrochronology, ENSO, rainfall.

\section{RESUMEN}

El ancho de los anillos de crecimiento y las fluctuaciones intra-anuales de densidad de la madera (IADF) contienen información sobre los factores ambientales que controlan el desarrollo de las especies forestales. En este trabajo se evaluaron los efectos del clima sobre el crecimiento radial y la formación de IADF en una plantación de Austrocedrus chilensis en Valdivia, Chile. Se calcularon la cronología media de crecimiento radial y la distribución anual de frecuencias de IADF de 20 árboles y se estudió su relación con la precipitación, la temperatura y los índices de El Niño Oscilación del Sur (ENSO) y Oscilación Antártica (AO) en el período 1975-2010. Se utilizaron análisis de correlación y regresión múltiple para estudiar la relación crecimiento-clima y la prueba $t$ de comparación de medias para contrastar diferencias entre años con y sin IADF. Se observó una relación inversa del crecimiento con la precipitación del verano previo y la temperatura máxima durante el período de crecimiento, evidenciando condiciones de estrés por exceso de precipitación y elevadas temperaturas estivales. Los años con máxima frecuencia de IADF en la madera de $A$. chilensis fueron aquellos en que se produjo un episodio El Niño, que en el área de estudio causa veranos secos y cálidos, seguidos por un mes de septiembre lluvioso. No se observaron relaciones significativas con AO. La interacción entre el clima local y ENSO ha controlado la formación de fluctuaciones intra-anuales de la densidad de la madera de A. chilensis en Valdivia.

Palabras clave: clima valdiviano, anatomía de la madera, dendrocronología, ENSO, pluviosidad.

\section{INTRODUCCIÓN}

Los anillos de crecimiento de los árboles contienen información sobre los factores que modulan la producción de madera en las especies forestales. La información proporcionada por el ancho de los anillos muestra una resolución anual, pero también es posible observar caracterís- ticas anatómicas peculiares dentro de un anillo de crecimiento que proporcionan información sobre eventos que han sucedido a una escala de tiempo sub-anual (Wimmer 2002). Estas características anatómicas permiten obtener información complementaria al ancho de los anillos y son especialmente valiosas cuando se intenta comprender la respuesta de las especies forestales a eventos como sequías 
(Hoffer y Tardif 2009, Edmondson 2010), las cuales pueden prolongarse por períodos de unos días a unos meses y normalmente tienen efectos nocivos sobre el vigor y la capacidad productiva de los árboles.

Entre las características anatómicas registradas en la madera se encuentran las fluctuaciones intra-anuales de densidad (IADF), también llamadas bandas intra-anuales $\mathrm{o}$ anillos falsos, los cuales constituyen un registro de alteraciones en la tasa de xilogénesis a lo largo de la estación de crecimiento. Las fluctuaciones de la densidad han sido estudiadas en diversas especies forestales, especialmente en coníferas (Rigling et al. 2001, Edmondson 2010). Estas bandas intra-anuales coinciden con una variación en la relación isotópica ${ }^{13} \mathrm{C} /{ }^{12} \mathrm{C}$ de la celulosa, indicando que están asociadas a respuestas plásticas a estreses fisiológicos (De Micco et al. 2007).

La madera de las coníferas está formada básicamente por traqueidas, las cuales presentan diámetros relativamente grandes y paredes celulares angostas en el leño temprano, pero menor lumen y paredes celulares más gruesas en el leño tardío. La distinción visual entre el leño temprano más claro, y el leño tardío más oscuro, permite diferenciar claramente los anillos anuales de crecimiento en la mayoría de las especies de coníferas, y por lo tanto facilita la identificación de las fluctuaciones de densidad (Olano et al. 2012). Por lo tanto, es posible realizar cronologías de frecuencias anuales de fluctuaciones en una población de árboles e identificar los factores climáticos que han condicionado su formación (Rigling et al. 2001, Rozas et al. 2011).

El ciprés de la cordillera (Austrocedrus chilensis (D. Don) Florin et Boutelje) es una especie de la familia Cupressaceae y una importante conífera de los bosques Andino-Patagónicos de Chile y Argentina. Es una especie longeva que puede superar los 1.400 años de edad, propia de ambientes xéricos sobre sustrato rocoso que se distribuye principalmente por las vertientes occidental y oriental de la Cordillera de Los Andes, en latitudes entre los $32^{\circ}$ $29^{\prime} \mathrm{S}$ y $43^{\circ} 38^{\prime} \mathrm{S}$ (Le Quesne et al. 2014). Su madera posee excelentes cualidades para la realización de estudios dendrocronológicos, puesto que forma anillos de crecimiento claramente definidos (Díaz-Vaz 1985), y su crecimiento anual está directamente relacionado con la disponibilidad hídrica (Le Quesne et al. 2014). Esta especie ha sido utilizada para la realización de reconstrucciones dendroclimáticas del régimen de precipitaciones y sequías (Le Quesne et al. 2006, Christie et al. 2011).

En el arboreto de la Universidad Austral de Chile en Isla Teja, Valdivia, existe una plantación de $A$. chilensis que se encuentra fuera del área de distribución natural de la especie para su conservación ex situ, en un ambiente mucho menos restrictivo que las condiciones ambientales en que habitualmente crece este árbol. El clima valdiviano es templado lluvioso con influencia mediterránea, con alta pluviosidad concentrada en el invierno, ausencia de una estación seca y temperaturas moderadas en verano (Gon-
zález-Reyes y Muñoz 2013). La precipitación en Valdivia está influenciada por dos teleconexiones de gran escala, El Niño Oscilación del Sur (ENSO) y Oscilación Antártica (AO) (González-Reyes y Muñoz 2013). La precipitación es menor en la fase cálida de ENSO tras eventos El Niño y mayor tras eventos La Niña (Montecinos y Aceituno 2003). La oscilación AO muestra una relación inversa con la precipitación de otoño y en los meses de febrero y marzo en Valdivia (González-Reyes y Muñoz 2013).

Considerando que no se dispone de conocimientos previos sobre los factores climáticos que influyen sobre el crecimiento y la formación de fluctuaciones de densidad en la madera de $A$. chilensis bajo el clima valdiviano, se plantearon dos objetivos de investigación: 1) evaluar los efectos del clima local y las teleconexiones ENSO y AO sobre el crecimiento radial, y 2) determinar los factores climáticos que facilitan la formación de fluctuaciones intra-anuales en la madera de $A$. chilensis. Las hipótesis son: 1) que el crecimiento radial en $A$. chilensis depende directamente de la disponibilidad de agua en forma de precipitación, y 2) que la formación de fluctuaciones de la densidad está asociada a la ocurrencia de períodos secos, la cual está determinada por la variación de ENSO y AO. Por lo tanto, en un periodo de baja precipitación el crecimiento anual de los árboles será menor y aumentará la probabilidad de formación de fluctuaciones de la densidad.

\section{MÉTODOS}

Área de estudio. El área de estudio se ubica en el arboreto de la Universidad Austral de Chile en Valdivia, Chile (39 $\left.48^{\prime} 51^{\prime \prime} \mathrm{S}, 73^{\circ} 14^{\prime} 45^{\prime \prime} \mathrm{O}\right)$. Los orígenes del arboreto datan del año 1971, cuando la Facultad de Ciencias Forestales ocupó un terreno de praderas y quebradas de bosque nativo para fines experimentales en el sector norte de la Isla Teja (Hechenleitner y Zamorano 2005). El arboreto tiene una superficie de 54 hectáreas y cuenta con una colección de 139 especies de plantas chilenas y 297 especies de plantas exóticas, constituyendo una de las mayores colecciones botánicas de Chile. La vegetación nativa comprende remanentes del bosque valdiviano lluvioso, dominado por ejemplares maduros de olivillo (Aextoxicon punctatum Ruiz et Pav.), coihue (Nothofagus dombeyi (Mirb.) Oerst.) y pellín (N. obliqua (Mirb.) Oerst.). Gran parte de la vegetación arbórea y arbustiva nativa existente en el arboreto se clasifica dentro del tipo forestal siempreverde con intolerantes emergentes, la que ocupa alrededor del $60 \%$ de la superficie total con cerca de 130 especies.

Suelo. El suelo se formó a partir de cenizas volcánicas antiguas, con predominio de la serie Correltúe (Andic Palehumult), con influencia en la superficie de cenizas volcánicas modernas de la serie Valdivia (Typic Hapludand). Estas cenizas volcánicas se depositaron sobre esquistos metamórficos, dando lugar al desarrollo de un suelo profundo, franco limoso, rico en materia orgánica y con alto 
contenido de nitrógeno total. Presenta bajos niveles de bases extraíbles, muy bajo contenido en fósforo disponible, fuertemente ácido con valores de $\mathrm{pH}$ en torno a 5, y alta saturación de aluminio intercambiable (Gerding et al. 2006).

Clima. Se utilizaron series de precipitación y temperatura mensuales registradas durante el periodo 1960-2010 en la estación meteorológica de Isla Teja, Valdivia, ubicada a una altitud de $9 \mathrm{~m}$ s.n.m. y aproximadamente a $1,4 \mathrm{~km}$ del sitio de estudio. La precipitación media anual fue de $2.310 \mathrm{~mm}$ y la temperatura media anual de $12,17^{\circ} \mathrm{C}$, con una temperatura media mínima de $7,95^{\circ} \mathrm{C}$, y máxima de $17,04{ }^{\circ} \mathrm{C}$. Los meses más húmedos fueron mayo, junio, julio y agosto, en que se superaron los $300 \mathrm{~mm}$ mensuales, mientras que los más secos fueron diciembre, enero, febrero y marzo, en que no se superaron los $100 \mathrm{~mm}$ mensuales (figura 1). Los meses más cálidos fueron diciembre, enero y febrero, con temperaturas medias superiores a $15^{\circ} \mathrm{C}$ y máximas superiores a $20^{\circ} \mathrm{C}$, mientras que los meses más fríos fueron junio, julio y agosto, con una temperatura media inferior a $10{ }^{\circ} \mathrm{C}$ (figura 1 ).

La serie de precipitación total anual no mostró una tendencia significativa durante el período 1960-2010, lo que indicó un valor de precipitación media aproximadamente constante (figura 2A). Se observó una gran variabilidad interanual de la precipitación total a partir de 1985, siendo destacables dos valores mínimos de 1.438 y $1.393 \mathrm{~mm}$ en 1988 y 1998, respectivamente, y un máximo de $3.149 \mathrm{~mm}$ en 2002 (figura 2A). Por otra parte, las temperaturas medias y máximas aumentaron significativamente durante el período 1960-2010, aumento especialmente pronunciado en el caso de las máximas, mientras que las temperaturas mínimas no mostraron una variación significativa a lo largo de ese período (figura 2B).

Las series de índices de teleconexiones climáticas para ENSO y AO se obtuvieron a partir de bases de datos de libre acceso en la Web. Se utilizaron los índices ENSO trimestral y AO mensual (NOAA 2015) y los datos bimensuales del Multivariate ENSO Index (MEI) (ESRL 2015).

Muestreo, preparación de las muestras y análisis dendrocronológicos. El muestreo dendrocronológico se realizó en una plantación de la especie $A$. chilensis establecida en el año 1973 a partir de semillas procedentes de Isla Victoria, Bariloche, Argentina. La plantación está compuesta por individuos coetáneos, de un tamaño similar y con un marco de plantación regular de $2 \times 2 \mathrm{~m}$. El diámetro (media $\pm \mathrm{SD}$ ) de los árboles fue $26,0 \pm 4,8 \mathrm{~cm}$ y la altura fue $15,5 \pm 2,0 \mathrm{~m}$. Los árboles de la plantación no mostraron síntomas de decaimiento ni afección por patógenos. En mayo de 2014 se tomaron uno o dos testigos de madera de $5 \mathrm{~mm}$ de diámetro por árbol de un total de 20 árboles mediante barrenos manuales de incremento. Un tamaño muestral de 20 árboles es considerado suficiente en estudios dendrocronológicos (Masiokas y Villalba 2004). Las condiciones existentes en una plantación homogénea minimizaron las posibles divergencias entre individuos debidas a edad, tamaño y nivel de competencia, factores que pueden ejercer un notable efecto sobre la variabilidad entre individuos en el crecimiento y la frecuencia de fluctuaciones de la densidad (Vieira et al. 2009).

Los testigos de A. chilensis colectados fueron preparados siguiendo los procedimientos dendrocronológicos convencionales, que incluyen su secado a temperatura ambiente y el montaje de las muestras en soportes de madera

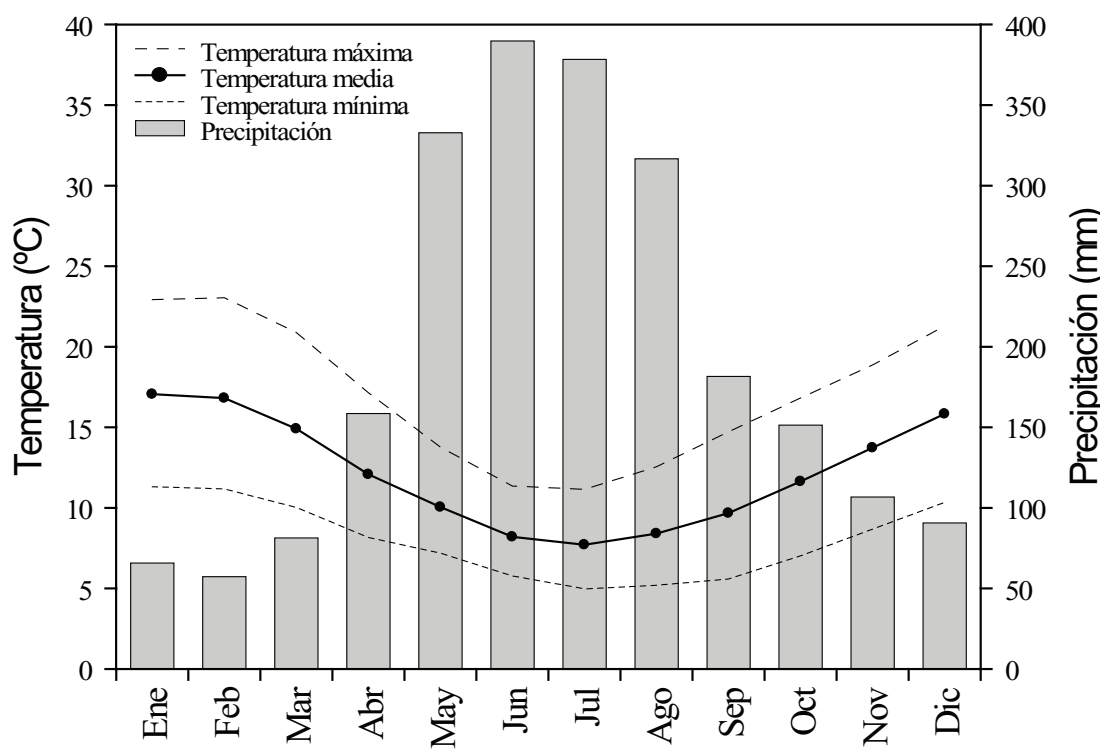

Figura 1. Diagrama climático de Isla Teja, Valdivia, período 1960-2010.

Climatic diagram of Isla Teja, Valdivia, period 1960-2010. 

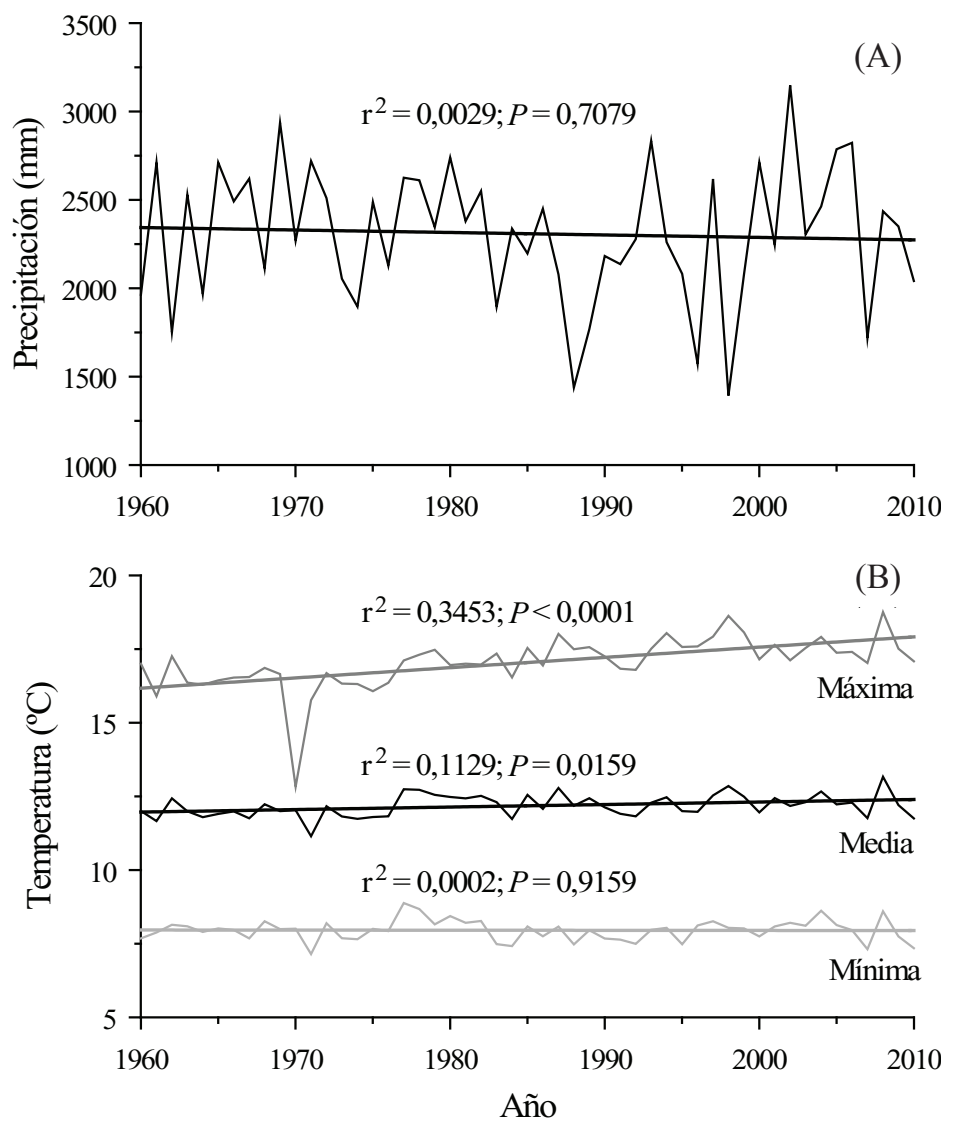

Figura 2. Tendencias de la precipitación total anual (A) y de las temperaturas máxima, media y mínima (B) en el período $1960-2010$. Se muestran los correspondientes ajustes lineares, los coeficientes de determinación $\left(r^{2}\right)$ y su correspondiente significación $(P)$.

Trends for total annual precipitation (A) and maximum, mean and minimum temperatures (B) in the period 1960-2010. Their linear fits and the corresponding coefficients of determination $\left(r^{2}\right)$ and statistical significance $(P)$ are shown.

acanalados. Las muestras fueron posteriormente preparadas con una lijadora manual utilizando granulometrías ascendentes entre 100 y 800 granos $\mathrm{cm}^{-2}$. Una vez lograda la perfecta visualización de los anillos de crecimiento, las muestras fueron fechadas bajo la lupa, asignándole un año calendario a cada anillo de crecimiento anual. El procedimiento de fechado siguió la convención para el hemisferio Sur que asigna a cada anillo anual el año de calendario en el cual se inició el crecimiento.

Las series individuales de ancho total de los anillos de crecimiento de los 20 árboles muestreados fueron medidas bajo aumento con una precisión de $0,001 \mathrm{~mm}$ utilizando una plataforma de medición Velmex. Para detectar errores de fechado debido a la presencia de falsos anillos o errores en el proceso de medición, las series de ancho de anillos se dataron visualmente y posteriormente la calidad del fechado fue chequeada utilizando el programa COFECHA (Grissino-Mayer 2001) y mediante comparación gráfica de las series individuales con la cronología de referencia para el sitio.

Las series brutas de ancho de anillos fueron promediadas anualmente para la obtención de una cronología media de crecimiento radial. El programa ARSTAN (Cook y Holmes 1996) se utilizó para elaborar la cronología estandarizada para el sitio de estudio. En esta cronología se eliminó la tendencia biológica de crecimiento y señales de posibles perturbaciones empleando un doble proceso de estandarización. Inicialmente se ajustaron curvas de tipo lineal o exponencial negativo y a los residuos resultantes se les aplicaron funciones de ajuste spline de 32 años, minimizándose las variaciones de ancho de anillos no comunes a todos los árboles de la plantación. Las series individuales estandarizadas se promediaron en una única cronología mediante una función de media robusta ponderada (Cook y Holmes 1996).

Identificación, registro y procesado de los datos de IADF. Los testigos de madera obtenidos de los 20 árboles muestreados, adecuadamente datados, medidos y cofechados, fueron examinados bajo la lupa para la identificación de las fluctuaciones de densidad. Al igual que en otras cupresáceas, el leño tardío de $A$. chilensis está muy poco desarrollado, constituyendo tan solo una pequeña proporción del ancho total del anillo de crecimiento (Olano et al. 
2012), y las IADF aparecen como bandas de traqueidas típicas del leño tardío inmersas en el leño temprano (Edmondson 2010). Las fluctuaciones de densidad se diferenciaron de los límites entre anillos por su transición gradual, al contrario que los auténticos límites que muestran una transición brusca (Wimmer 2002). Los límites entre anillos mostraron una transición brusca en tamaño celular y grosor de las paredes celulares entre el leño tardío de un año y el leño temprano del año siguiente, mientras que las fluctuaciones de densidad mostraron una transición gradual con la madera adyacente, tanto al inicio como al final de la fluctuación (figura 3).

Se registró anualmente la presencia de fluctuaciones de la densidad en los testigos de madera. La frecuencia relativa de IADF fue calculada usando la ecuación [1]. La heterogeneidad de varianza inducida por el número variable de muestras por año fue corregida mediante el cálculo de la frecuencia estabilizada de fluctuaciones $\left(F_{\text {estab }}\right)$ mediante la ecuación propuesta por Rigling et al. (2001) [2]:

$$
\begin{gathered}
F=n_{x} / N \\
F_{\text {estab }}=F \times N^{0,5}
\end{gathered}
$$

donde $F=$ frecuencia relativa de IADF, $n_{x}=$ número de muestras con una fluctuación en el año $x$ y $N=$ número total de muestras representadas en ese año.

La distribución anual de frecuencias estabilizadas de IADF fue utilizada para el cálculo de relaciones estadísticas con las cronologías media y estandarizada de ancho de anillos y con las series climáticas.

Cálculo de las relaciones con clima. El cálculo de las relaciones estadísticas entre las series climáticas, la cronología estandarizada de anchos de anillos y la distribución de
$F_{\text {estab }}$ de fluctuaciones de densidad se realizó en 1975-2010, período en que estuvieron representados al menos cinco árboles en las series dendrocronológicas y en que se dispuso de series de datos climáticos. El período con al menos cinco árboles es utilizado habitualmente y considerado suficiente en estudios dendrocronológicos (Wimmer 2002). La correlación de Pearson fue usada para calcular la relación entre la distribución de $F_{\text {estab }}$ y las cronologías media y estandarizada de ancho de anillos. También se utilizó análisis de correlación para identificar las variables climáticas que presentaron una relación estadísticamente significativa con la cronología estandarizada y la distribución de frecuencias estabilizadas de fluctuaciones de densidad. Se realizaron análisis de regresión lineal múltiple para calcular las relaciones existentes entre las series temporales de variables climáticas y las series dendrocronológicas, tanto para la cronología estandarizada como para la distribución de frecuencias estabilizadas de IADF. La existencia de diferencias en los registros climáticos entre años sin fluctuaciones de densidad de la madera y años con elevada frecuencia de fluctuaciones de densidad, se contrastó mediante la prueba $t$ para comparación de medias. Finalmente, las desviaciones de los datos climáticos medios para los años con mayor $F_{\text {estab }}$, respecto de las medias climáticas en el período 1975-2010, se calcularon para comprobar la correspondencia entre la formación de fluctuaciones de densidad y la ocurrencia de eventos climáticos que se desvíen de lo esperado bajo el clima de Valdivia.

\section{RESULTADOS}

Cronología de ancho de anillos y relaciones con clima. $\mathrm{La}$ señal común entre árboles y series de ancho de anillos de crecimiento de $A$. chilensis fue muy elevada. La proporción de varianza explicada por el primer vector propio fue de $55,8 \%$, la correlación media entre árboles de 0,515 , y la correlación entre series de crecimiento de 0,522 , indicando una gran

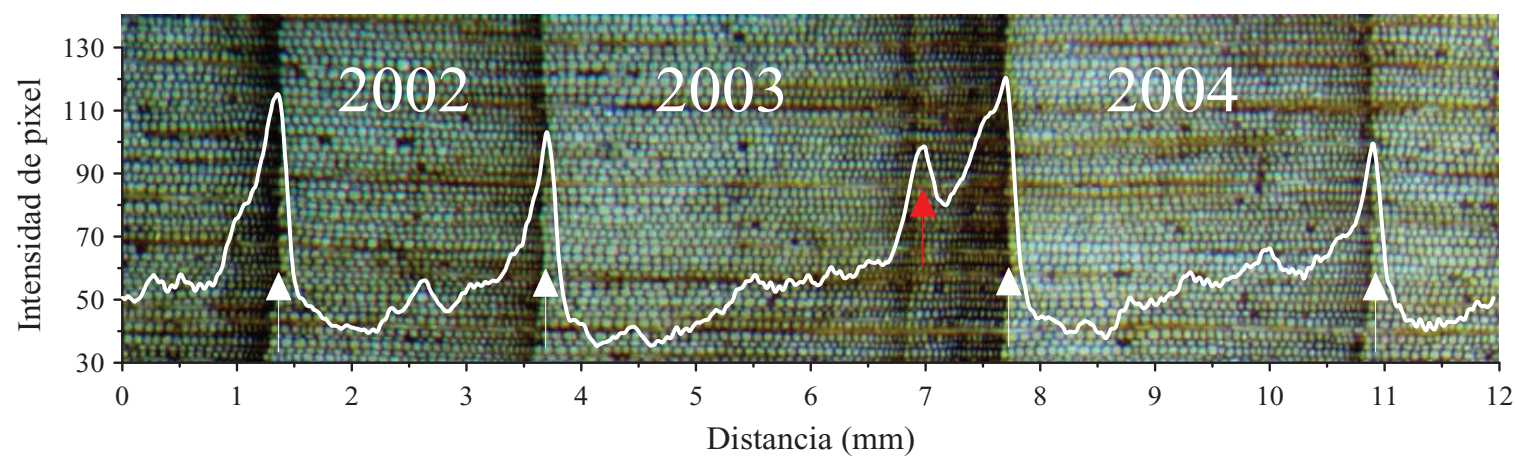

Figura 3. Anillos de crecimiento de A. chilensis con su correspondiente perfil de intensidad de los pixeles. Las flechas blancas señalan los límites entre anillos de crecimiento, la flecha roja señala una fluctuación intra-anual de la densidad o falso anillo. La escala de intensidad de pixel está basada en los valores de gris de la imagen, según el programa ImageJ 1.49.

Tree rings of $A$. chilensis with their profile of pixel intensities. White arrows indicate the limits between tree rings, the red arrow indicates an intrannual density fluctuation or false ring. Pixel intensities are scaled based on the gray values, according to the Image J 1.49 software. 
correspondencia entre el crecimiento de los árboles muestreados. La cronología estandarizada de ancho de anillos de crecimiento, aunque de corta longitud, mostró una excelente calidad. La relación señal-ruido fue de 19,65 y la señal expresada de la población fue de 0,952 , lo cual indica una notable señal común compartida entre las series de crecimiento que componen la cronología. Los años que mostraron mayores crecimientos fueron 1979, 1989, 1992, 2000, 2009 y 2010, mientras que en los años 1981, 1983, 1995, 2001, 2007 y 2013 se registraron los menores crecimientos (figura 4).

La cronología estandarizada de ancho de anillos de crecimiento mostró correlaciones negativas significativas con la precipitación de enero del año de crecimiento $(r=-0,481, P=0,002)$ y con las temperaturas máxima $(r=-0,570, P=0,000)$ y media $(r=-0,472, P=0,003)$ de diciembre del año de crecimiento. El modelo de regresión múltiple reveló que las dos últimas variables climáticas mostraron un efecto redundante y que el crecimiento de los anillos de $A$. chilensis estuvo determinado por una combinación lineal entre la precipitación de enero y la temperatura máxima de diciembre, las cuales explicaron en conjunto un $48,8 \%$ de la varianza del crecimiento (cuadro 1).

Frecuencia de fluctuaciones de densidad. Las IADF se localizaron, en todos los casos, en el tercio final de los anillos de crecimiento. La proporción de testigos de madera que mostraron IADF fue del $100 \%$, variando entre un mínimo de 7 y un máximo de 17 fluctuaciones de densidad por testigo. En el período 1975-2013 se registraron un total de 256 fluctuaciones de densidad, lo que significó que el $31,8 \%$ de los anillos disponibles en ese período mostraron una fluctuación de densidad. La distribución anual de frecuencias de IADF no mostró ninguna relación con el ancho de los anillos de crecimiento (figura 4), tanto con las series de ancho bruto $(r=0,175, P=0,287)$ como con las series residuales que componen la cronología estandariza-
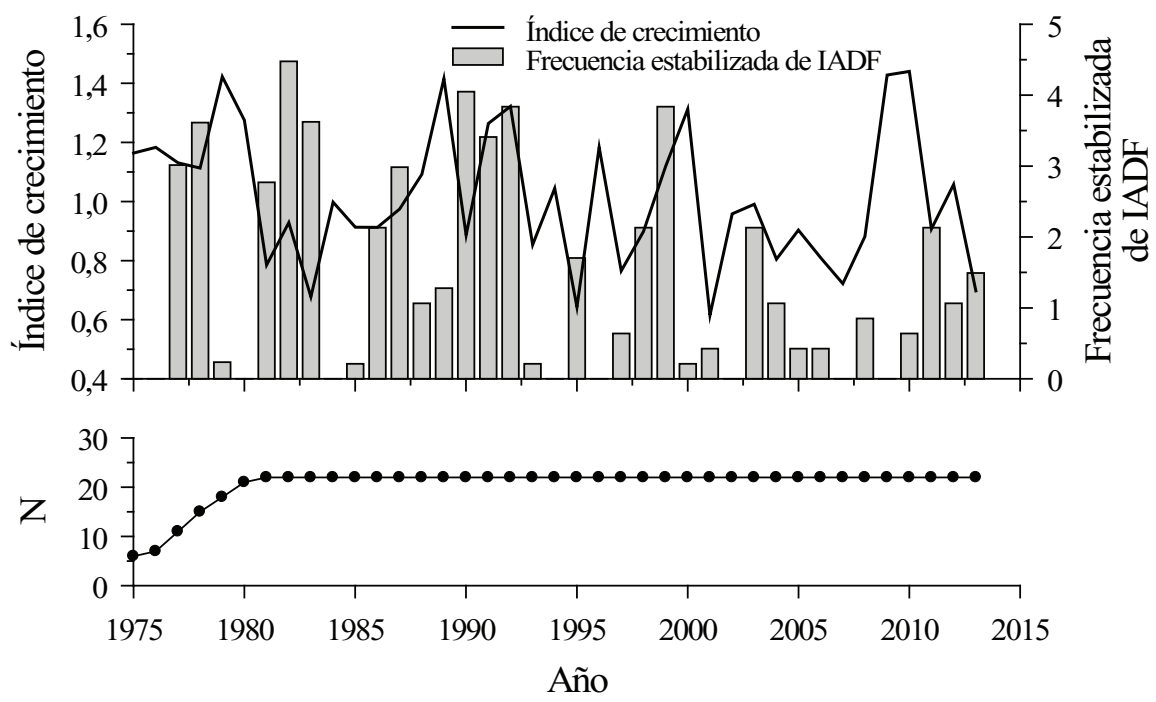

Figura 4. Cronología de índices de crecimiento anual estandarizados de $A$. chilensis y variación anual de las frecuencias estabilizadas de fluctuaciones intra-anuales de densidad de la madera y el tamaño muestral $(\mathrm{N})$ de series de crecimiento estudiadas.

Chronologies of standardized tree-ring growth indices for A. chilensis and the annual variation of stabilized frequencies of intrannual wood density fluctuations, and the sample size $(\mathrm{N})$ of studied tree-ring series.

Cuadro 1. Regresiones lineales múltiples con las variables climáticas que condicionan significativamente el crecimiento y la formación de IADF.

Multiple linear regressions with the most relevant climatic variables on tree growth and IADFs formation.

\begin{tabular}{lccclcrrr}
\hline Variable dependiente & $r^{2}$ del modelo & $F$ del modelo & $P$ del modelo & Predictor climático ${ }^{\text {a }}$ & $\beta$ & $t$ & $P$ \\
\hline \multirow{2}{*}{ Cronología de crecimiento } & 0,488 & 15,75 & $<0,001$ & Diciembre Tmax & $-0,489$ & $-3,86$ & $<0,001$ \\
& & & & Enero Prec & $-0,412$ & $-3,25$ & 0,003 \\
\hline \multirow{2}{*}{$F_{\text {estab }}$ de IADF } & 0,405 & 11,22 & $<0.001$ & Septiembre Prec & 0,470 & 3,37 & 0,002 \\
& & & & Febrero-Marzo MEI & 0,318 & 2,27 & 0,029 \\
\hline
\end{tabular}

a Tmax: temperatura máxima, Prec: precipitación, MEI: índice multivariable ENSO. 
da de crecimiento $(r=-0,100, P=0,545)$. Lo cual sugirió que los eventos causantes de la formación de IADF fueron independientes de los factores climáticos que controlaron el crecimiento radial. Los años que mostraron las mayores frecuencias de IADF fueron 1977, 1978, 1982, 1983, 1990, 1991, 1992 y 1999, mientras que no se observó ninguna fluctuación de densidad en los años 1975, 1976, 1980, 1984, 1994, 1996, 2002, 2007 y 2009 (figura 4).

Condicionantes climáticos de la formación de IADF. La distribución anual de frecuencias estabilizadas de IADF mostró correlaciones significativas positivas con la precipitación de septiembre $(r=0,558, P=0,000)$, con el MEI de febrero-marzo $(r=0,448, P=0,005)$ y con el índice ENSO desde noviembre-enero a febrero-abril $(r=0,378$ a 0,388 , $P=0,019$ a 0,016$)$. El modelo de regresión múltiple reveló que la formación de IADF en la madera de $A$. chilensis estuvo relacionada con la combinación de una abundante precipitación en septiembre y un índice MEI elevado en febrero y marzo, explicando ambos factores en conjunto un 40,5\% de la varianza de la frecuencia de fluctuaciones de densidad (cuadro 1). La distribución de frecuencias de IADF y la cronología estandarizada del crecimiento no mostraron una relación estadística significativa con los índices de la AO.

La comparación entre medias de los factores climáticos mensuales para los años $\sin \operatorname{IADF}\left(F_{\text {estab }}=0\right)$ y con una elevada frecuencia de IADF $\left(F_{\text {estab }}>2\right)$, durante el año previo al crecimiento y el propio año de crecimiento, mostró que sólo la precipitación de septiembre fue significativamente mayor $(t=2,931 ; P=0,009)$ en los años con elevada frecuencia de fluctuaciones de la densidad que en los años sin fluctuaciones (figura 5A). Las desviaciones
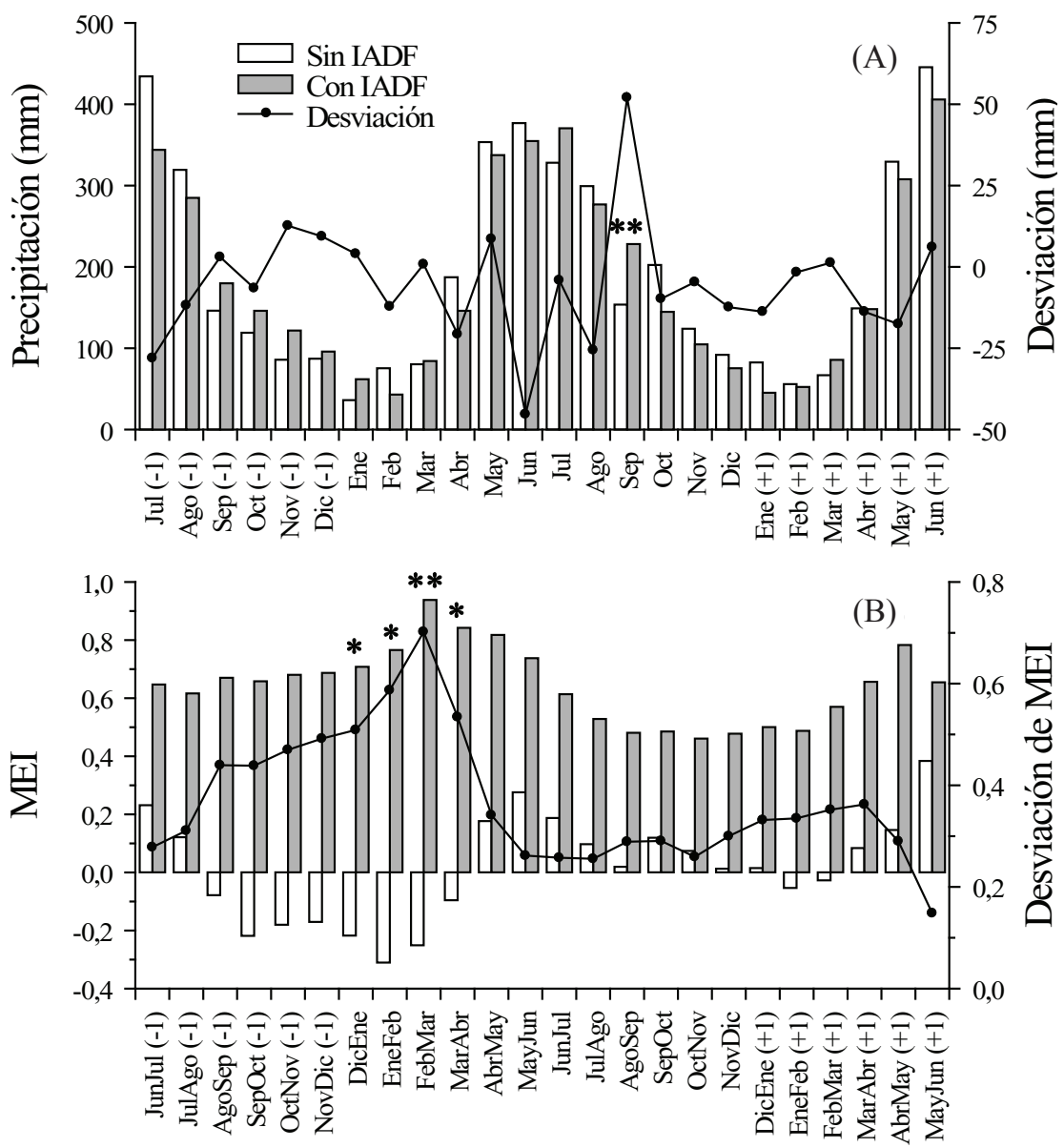

Figura 5. Comparación de medias y desviaciones de la precipitación (A) y el MEI (B) para años sin IADF (1975, 1976, 1980, 1984, 1994, 1996, 2002, 2007 y 2009) y con elevada frecuencia de IADF (1977, 1978, 1981, 1982, 1983, 1986, 1987, 1990, 1991, 1992, 1998, 1999 y 2003). Las desviaciones de precipitación y MEI se han calculado respecto a las medias mensuales del período 1975-2010. Se señalan las medias significativamente diferentes, de acuerdo con la prueba $t(*=P<0.05 ; * *=P<0,01) .(-1)$ y $(+1)$ indican el año previo y siguiente al de inicio del crecimiento.

Comparison of means and deviations for precipitation (A) and MEI (B) for years without IADFs (1975, 1976, 1980, 1984, 1994, 1996, 2002, 2007 and 2009) and with high IADFs frequency $(1977,1978,1981,1982,1983,1986,1987,1990,1991,1992,1998,1999$ and 2003). Deviations of precipitation and MEI from the period 1975-2010 were calculated. Significantly different means, according to $t$ test, are shown $(*=P<0.05 ; * *=$ $P<0.01)$. $(-1)$ and $(+1)$ indicate the years before and after those year in which growth started. 
respecto a la media del período 1975-2010 mostraron que las fluctuaciones de densidad se produjeron en años con una precipitación de septiembre $52 \mathrm{~mm}$ superior a la media. En el caso de las temperaturas máximas, no existieron diferencias significativas entre los años sin fluctuaciones y los años con elevada frecuencia de fluctuaciones $(P>$ 0,05 en todos los casos). En el caso del MEI, sin embargo, los índices correspondientes a los meses desde diciembre a abril previos al crecimiento difirieron entre años (DicEne MEI: $t=2,241 ; P=0,036$; EneFeb MEI: $t=2,717$; $P=0,014$; FebMar MEI: $t=3,133 ; P=0,005$; MarAbr MEI: $t=2,341 ; P=0,030$ ), siendo mayores en los años con elevada frecuencia de fluctuaciones que en los años sin fluctuaciones (figura 5B). De hecho, las desviaciones respecto a la media del período 1975-2010 mostraron que las fluctuaciones de densidad se produjeron en años con índices MEI superiores a la media entre agosto-septiembre y marzo-abril, pero especialmente elevado en febrero-marzo previos a la estación de crecimiento, con una desviación de 0,70 superior a la media.

Fue evidente una correspondencia temporal entre la distribución de frecuencias de IADF en la madera de A. chilensis y la variación temporal del índice ENSO. Los años en que se produjo un evento El Niño (índice ENSO $>0,5)$, o el año inmediatamente posterior, mostraron un aumento de la frecuencia de IADF en los anillos de crecimiento, mientras que tras los períodos La Niña (índice ENSO $<-0,5)$ generalmente disminuyó la frecuencia de IADF (figura 6).

\section{DISCUSIÓN}

Relación entre crecimiento radial y clima. Los resultados obtenidos evidencian que el crecimiento de $A$. chilensis en el área de estudio es mayor en los años con escasa precipitación en enero previo a la estación de crecimiento. En su área de distribución natural, el crecimiento de A. chilensis típicamente muestra una respuesta positiva a la precipitación, de forma más intensa durante la estación húmeda previa al inicio de su actividad cambial (Le Quesne et al. 2006, Mundo et al. 2010). Puesto que durante estos meses la precipitación es muy abundante en Valdivia, probablemente no existe una limitación hídrica para la especie, por lo que no existe una respuesta positiva al volumen de lluvia caída durante el invierno. Hay que tener en cuenta que esta especie crece de forma natural en Chile en ambientes de montaña, sobre suelos muy poco desarrollados o en grietas de rocas, bajo un clima seco en que las escasas precipitaciones se concentran en junio, julio y agosto, con algunas lluvias ocasionales en mayo y septiembre, y un extenso período seco entre octubre y abril (Le Quesne et al. 2014). En las condiciones de elevada pluviosidad de Valdivia, esta especie podría necesitar un verano más seco que lo esperable en esta localidad. De ahí la relación negativa del crecimiento con la precipitación de enero, el segundo mes más seco del año.

Este resultado podría interpretarse como un efecto negativo de la precipitación sobre el crecimiento en un período en que los árboles deberían sufrir una marcada sequía

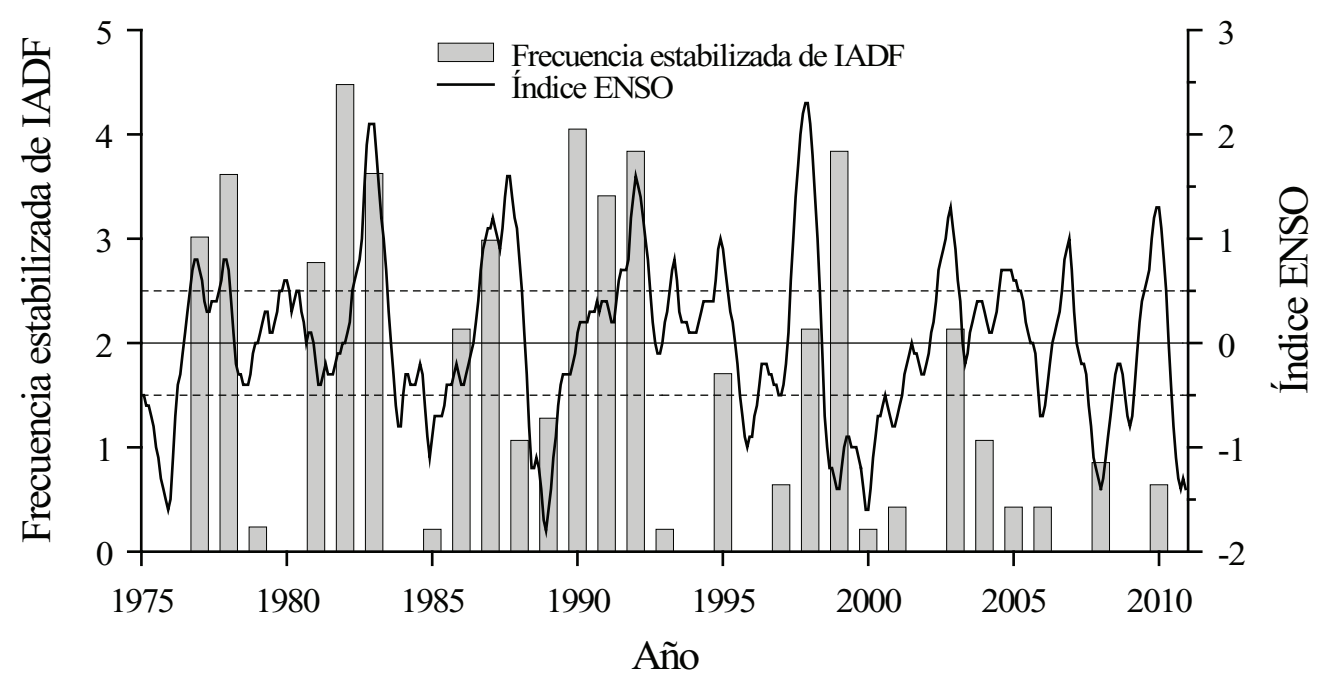

Figura 6. Correspondencia temporal entre la distribución anual de frecuencias estabilizadas de IADF en la madera de $A$. chilensis y el índice ENSO. Las líneas segmentadas indican los valores 0,5 y -0,5 de ENSO. Valores de ENSO > 0,5 indican eventos "El Niño", valores de ENSO $<-0,5$ indican eventos "La Niña".

Correspondence between yearly distribution of the stabilized frequency of IADFs in A. chilensis wood and the MEI index. Dashed lines indicate 0.5 and -0.5 ENSO values. ENSO values $>0.5$ indicate "El Niño" events, ENSO values $<-0.5$ indicate "La Niña" events. 
en su área de distribución natural. Se ha constatado que el llamado "mal del ciprés", un proceso de decaimiento y muerte masiva de esta especie registrado desde mediados del siglo XX en diversas partes de su área de distribución, se produce con mayor probabilidad en ambientes húmedos sobre suelos con textura fina, abundancia de arcilla, escasa pendiente, poco drenaje y proximidad a cursos de agua (La Manna et al. 2008). Aunque el estado sanitario de la plantación estudiada es bueno, sin síntomas evidentes de afección por el "mal del ciprés", se puede plantear la hipótesis de que mayor humedad durante el año previo al crecimiento aumentaría la actividad del hongo Phytophthora austrocedrae Greslebin et al., 2007, causante de la infección, produciendo daños en las raíces que se manifestarían como un menor crecimiento de los árboles el siguiente año. Para confirmar esta hipótesis sería necesario demostrar que efectivamente la plantación estudiada está infectada por el hongo patógeno, pero este análisis excede los objetivos planteados en esta investigación.

A los factores edáficos habría que añadir los factores climáticos como generadores de estrés y limitantes del crecimiento. Existen evidencias de que los síntomas de decaimiento de esta especie también son más frecuentes en áreas con elevada precipitación y baja altitud (Baccalá et al. 1998). Además, el crecimiento de los árboles en condiciones hídricas no limitantes puede mostrar una respuesta negativa a la precipitación, como ha sido observado en coníferas bajo clima húmedo (Mäkinen et al. 2002, Rozas et al.2013). Esta respuesta puede atribuirse a las condiciones típicas de los climas lluviosos, en que la abundante nubosidad reduce considerablemente la radiación solar, la tasa fotosintética, la asimilación de carbohidratos y el crecimiento de los árboles (Min y Wang 2008). Probablemente este efecto es acusado en A. chilensis bajo climas lluviosos, ya que la especie está adaptada a condiciones de montaña con abundante radiación, una marcada estación seca y suelos con muy buen drenaje.

Los resultados también muestran que el crecimiento de $A$. chilensis en Valdivia está limitado por las elevadas temperaturas diarias en diciembre, durante la estación de crecimiento. Esta respuesta es coincidente con un efecto negativo de las elevadas temperaturas de noviembre y diciembre sobre el crecimiento de esta especie en el norte de la Patagonia argentina (Mundo et al. 2010). La respuesta observada es equivalente a las respuestas negativas a la temperatura de verano evidenciadas por trabajos en coníferas del hemisferio norte (Vieira et al. 2009, Rozas et al. 2013). Sería interpretable como una limitación del crecimiento cuando ocurren temperaturas estivales elevadas causantes de estrés hídrico, probablemente superiores a las cuales la especie está adaptada en los ambientes de montaña. La pronunciada tendencia ascendente de las temperaturas máximas registradas en Valdivia durante las últimas décadas (figura 2B), implicaría que este efecto negativo de la temperatura máxima del verano sobre el crecimiento de A. chilensis podría acentuarse en el futuro.
Efectos del clima local y ENSO sobre la formación de $I A D F$. Los años con máxima frecuencia de IADF en la madera de $A$. chilensis son aquellos en que el índice MEI del verano previo al crecimiento es elevado, precedido o coincidente con un episodio El Niño, y el mes de septiembre del año de crecimiento es lluvioso. Es necesario señalar que ambas variables climáticas condicionantes de la formación de IADF, la precipitación de septiembre y el índice MEI de febrero-marzo, son independientes entre sí $(r=0,276, P=0,103)$. Por lo tanto, no existe una relación de dependencia directa entre la precipitación al inicio de la estación de crecimiento y los índices ENSO durante el verano previo al crecimiento. Septiembre es el mes en que se inicia el crecimiento de $A$. chilensis, el inicio de la primavera y de la actividad cambial (Le Quesne et al. 2006). Este resultado aparentemente es contradictorio con las causas climáticas habituales causantes de las fluctuaciones de densidad en coníferas del hemisferio norte, las cuales normalmente dependen positivamente de la precipitación en agosto-octubre, al final de la estación de crecimiento (Vieira et al. 2009, Rozas et al. 2011). Es decir, las lluvias que suceden eventualmente al final del verano e inicio del otoño, reactivan la actividad cambial cuando esta ya ha cesado. En el caso de A. chilensis en Valdivia, esta relación no es equivalente, ya que son las lluvias de septiembre, al inicio de la estación de crecimiento, las que propician la formación de una fluctuación de densidad en la madera.

La interpretación de este resultado hay que realizarla en conjunción con el efecto positivo de ENSO sobre la formación de fluctuaciones de densidad. Los años El Niño coinciden en el área de estudio con un verano seco y cálido, lo que podría causar una limitación hídrica en comparación con años La Niña, con veranos más templados y húmedos (González-Reyes y Muñoz 2013). En la latitud en que se encuentra Valdivia, entre los $38^{\circ}$ y $41^{\circ} \mathrm{S}$, es habitual un déficit de precipitación durante el verano (enero-febreromarzo) siguiente a un episodio El Niño, mientras que tras un episodio La Niña el verano es más lluvioso (Montecinos y Aceituno 2003). Aunque la especie $A$. chilensis habita normalmente en ambientes mucho más restrictivos que el existente en el arboreto de Valdivia, probablemente los árboles estudiados presentan una adaptación al ambiente local, de forma tal que pueden experimentar cierto estrés hídrico cuando se produce una sequía estival durante el año previo al crecimiento. Tras una sequía estival causada por ENSO, el crecimiento de esta especie podría detenerse antes de tiempo, mientras que las condiciones lluviosas en el septiembre siguiente podrían causar una posterior reactivación de la actividad cambial, lo que facilitaría la formación de una banda intra-anual.

La influencia de una teleconexión climática de gran escala sobre el clima local moduló la formación de fluctuaciones intra-anuales en la densidad de la madera de $A$. chilensis en Valdivia. En un estudio previo con la especie Pinus pinaster Ait. en el norte de España, el patrón del Atlántico Este (EA) controló fuertemente la temperatura 
de invierno y el balance hídrico en primavera, los cuales a su vez facilitaron la formación de IADF en la madera (Rozas et al. 2011). Sin embargo, el patrón de formación de IADF observado en $A$. chilensis no guarda una relación con otros patrones observados en especies similares o en áreas geográficas próximas. En el caso de la especie Juniperus virginiana $\mathrm{L}$. en clima semiárido, las fluctuaciones de densidad se forman en años en que se produjo una sequía combinada con una ola de calor durante la estación de crecimiento (Edmondson 2010). También muestra un comportamiento similar la especie Nothofagus pumilio (Poepp. et Endl.) Krasser en el sur de la Patagonia argentina, en que las IADF están asociadas a precipitaciones por debajo del promedio desde octubre a diciembre, combinado con temperaturas por encima del promedio desde noviembre a marzo (Masiokas y Villalba 2004). Esta disparidad sugiere que probablemente existe un fuerte componente regional relacionado con las condiciones climáticas locales, y su dependencia de forzantes climáticos de gran escala, en la combinación de factores ambientales desencadenantes de un estrés fisiológico suficientemente intenso que cause la formación de fluctuaciones de densidad en la madera.

\section{CONCLUSIONES}

Se rechaza la primera hipótesis de una relación directa entre precipitación y crecimiento, la cual es habitual para esta especie en su área de distribución bajo condiciones naturales pero no en la plantación de Valdivia. Este trabajo reveló que el crecimiento radial de A. chilensis en Valdivia está inversamente relacionado con la precipitación del año previo al período de crecimiento y la temperatura máxima en el verano del período de crecimiento. Estas respuestas podrían interpretarse a base de las condiciones climáticas locales, con ausencia de una estación seca, abundante nubosidad y existencia de un cierto estrés térmico estival, condiciones a que la especie no está sometida en su área de distribución natural. Los años con mayor probabilidad de formación de IADF coincidieron con eventos El Niño durante el verano previo al crecimiento, seguidos por un mes de septiembre lluvioso. Se acepta parcialmente la segunda hipótesis, ya que en el área de estudio el verano siguiente a un episodio El Niño es seco y una reducción de la precipitación causada por ENSO seguida de una primavera lluviosa facilitaría la formación de fluctuaciones intra-anuales de densidad. Sin embargo, no se observó ninguna relación entre AO y la frecuencia de IADF. Estas evidencias sugieren que la combinación entre el clima local y la variación a gran escala de ENSO habrían controlado en gran medida los procesos fisiológicos que determinaron la formación de fluctuaciones intra-anuales de la densidad de la madera de A. chilensis en Valdivia. Considerando los resultados obtenidos, se plantea la necesidad de realizar investigaciones dendrocronológicas adicionales en un ámbito geográfico y ambiental más amplio con el propósito de obtener un conocimiento más completo sobre la variabilidad de las respuestas del crecimiento y la formación de IADF en A. chilensis, tanto en plantaciones fuera de su área de distribución natural como en poblaciones naturales.

\section{AGRADECIMIENTOS}

Agradecemos la colaboración de Robert Brümmer, quien proporcionó las series de datos climáticos registrados en la estación meteorológica de Isla Teja, Valdivia. Esta investigación fue financiada por el proyecto "High Resolution Hydroclimate Variability in the Central Andes of Chile and Argentina during the Last Millennium: a Tree-Ring and Modelling Perspective" (FONDECYT 1121106). La estadía de V. Rozas en la Universidad Austral de Chile, Valdivia, fue financiada por CONICYT mediante el Concurso Nacional "Atracción de Capital Humano Avanzado del Extranjero, Modalidad Estadías Cortas (MEC)" (Código 80130061).

\section{REFERENCIAS}

Baccalá N, P Rosso, M Havrylenko. 1998. Austrocedrus chilensis mortality in the Nahuel Huapi National Park (Argentina). Forest Ecology and Management 109: 261-269.

Christie DA, J Boninsegna, M Cleaveland, A Lara, C Le Quesne, M Morales, M Mudelsee, D Stahle, R Villalba. 2011. Aridity changes in the Temperate-Mediterranean transition of the Andes since AD 1346 reconstructed from tree-rings. Climate Dynamics 36: 1505-1521.

Cook ER, RL Holmes. 1996. Guide for computer program ARSTAN. In Grissino-Mayer HD, RL Holmes, HC Fritts eds. The international tree ring data bank program library version 2.0 user's manual. Tucson, AZ, USA. Laboratory of Tree-Ring Research, University of Arizona. p. 75-87.

De Micco V, M Saurer, G Aronne, R Tognetti, P Cherubini. 2007. Variations of wood anatomy and $\delta 13 \mathrm{C}$ within-tree rings of coastal Pinus pinaster showing intra-annual density fluctuations. IAWA Journal 28: 61-74.

Díaz-Vaz JE. 1985. Austrocedrus chilensis, descripción anatómica. Bosque 6(1): 49-50.

Edmondson JR. 2010. The meteorological significance of false rings in eastern redcedar (Juniperus virginiana L.) from the southern Great Plains, U.S.A. Tree-Ring Research 66: 19-33.

ESRL (Earth System Research Laboratory, US) 2015. Multivariate ENSO Index. Consultado 5 ago. 2015. Disponible en http://www.esrl.noaa.gov/psd/enso/mei/

Gerding V, E Geldres, JA Moya. 2006. Diagnóstico del desarrollo de Pinus massoniana y Pinus brutia establecidos en el arboreto de la Universidad Austral de Chile, Valdivia. Bosque 27(1): 57-63.

González-Reyes A, AA Muñoz. 2013. Cambios en la precipitación de la ciudad de Valdivia (Chile) durante los últimos 150 años. Bosque 34(2): 191-200.

Grissino-Mayer HD. 2001. Evaluating crossdating accuracy: a manual and tutorial for the computer program COFECHA. Tree-Ring Research 57: 205-221.

Hechenleitner P, C Zamorano. 2005. Arboretum de la Universidad Austral de Chile: un modelo de conservación integral para nuestro país. Revista Chagual 3: 41-48. 
Hoffer M, JC Tardif. 2009. False rings in jack pine and black spruce trees from eastern Manitoba as indicators of dry summers. Canadian Journal of Forest Research 39: 1722-1736.

La Manna L, SD Matteucci, T Kitzberger. 2008. Abiotic factors related to the incidence of the Austrocedrus chilensis disease syndrome at a landscape scale. Forest Ecology and Management 256: 1087-1095.

Le Quesne C, D Stahle, M Cleaveland, MD Therrell, JC Aravena, J Barichivich. 2006. Ancient Austrocedrus tree-ring chronologies used to reconstruct central Chile precipitation variability from a.d. 1200 to 2000. Journal of Climate 19: 5731-5744.

Le Quesne C, M Rojas, D Christie. 2014. Anillos de crecimiento de Austrocedrus chilensis: un archivo natural del cambio climático. Revista Chagual 12: 31-35.

Mäkinen H, P Nöjd, HP Kahle, U Neumann, B Tveite, K Mielikäinen, H Röhle, H Spiecker. 2002. Radial growth variation of Norway spruce (Picea abies (L.) Karst.) across latitudinal and altitudinal gradients in central and northern Europe. Forest Ecology and Management 174: 233-249.

Masiokas M, R Villalba. 2004. Climatic significance of intraannual bands in the wood of Nothofagus pumilio in southern Patagonia. Trees 18: 696-704.

Min Q, S Wang. 2008. Clouds modulate terrestrial carbon uptake in a midlatitude hardwood forest. Geophysical Research Letters 35. DOI: 10.1029/2007GL032398.

Montecinos A, P Aceituno. 2003. Seasonality of the ENSO-related rainfall variability in central Chile and associated circulation anomalies. Journal of Climate 16: 281-296.

Mundo IA, VA El Mujtar, MH Perdomo, LA Gallo, R Villalba,
MD Barrera. 2010. Austrocedrus chilensis growth decline in relation to drought events in northern Patagonia, Argentina. Trees 24: 561-570.

NOAA (National Oceanic and Atmospheric Administration, US) 2015. ENSO trimestral y AO mensual. Consultado 5 ago. 2015. Disponible en http://www.cpc.ncep.noaa.gov/products/precip/CWlink/

Olano JM, M Eugenio, AI García-Cervigón, M Folch, V Rozas. 2012. Quantitative tracheid anatomy reveals a complex environmental control of wood structure in continental Mediterranean climate. International Journal of Plant Sciences 173: 137-149.

Rigling A, PO Waldner, T Forster, OU Bräker, A Pouttu. 2001. Ecological interpretation of tree-ring width and intraannual density fluctuations in Pinus sylvestris on dry sites in the central Alps and Siberia. Canadian Journal of Forest Research 31: 18-31.

Rozas V, I García-González, R Zas. 2011. Climatic control of intra-annual wood density fluctuations of Pinus pinaster in NW Spain. Trees 25: 443-453.

Rozas V, I García-González, G Pérez-de-Lis, JR Arévalo. 2013. Local and large-scale climatic factors controlling tree-ring growth of Pinus canariensis on an oceanic island. Climate Research 56: 197-207.

Vieira J, F Campelo, C Nabais. 2009. Age-dependent responses of tree-ring growth and intra-annual density fluctuations of Pinus pinaster to Mediterranean climate. Trees 23: 257-265.

Wimmer R. 2002. Wood anatomical features in tree-rings as indicators of environmental change. Dendrochronologia 20: 21-36.

Recibido: 22.09.15

Aceptado: 27.04.16 
